\title{
Sensor Actor Network Modeling utilizing the Holonic Architectural Framework
}

\author{
Christopher Chiu, Zenon Chaczko, Perez Moses
}

\begin{abstract}
This paper discusses the results of utilizing advanced EKM modeling techniques to manage Sensor-Actor networks (SANETs) based upon the Holonic Architectural Framework. EKMs allow a quantitative analysis of an algorithmic artificial neural network process by using an indirect-mapping EKM to self-organize from a given input space to administer SANET routing and clustering functions with a control parameter space. Results demonstrate that in comparison to linear approximation techniques, indirect mapping with EKMs provide fluid control and feedback mechanisms by operating in a continuous sensory control space - thus enabling interactive detection and optimization of events in real-time environments.
\end{abstract}

Keywords - Extended Kohonen Maps (EKM), Sensor Actor Networks (SANET), Wireless Sensor Networks (WSN), SANET Middleware, POE Classification Model, Holonic Architecture

\section{THE NECESSITY FOR SANET MIDDLEWARE}

$\mathrm{C}$ URRENT SANET middleware technologies commonly utilize commercial of-the-shelf (COTS) products, such as Microsoft's .NET Remoting, Sun's Remote Method Invocation (RMI), and Object Management Group's (OMG) Common Object Request Broker (CORBA) [14]. These solutions are being used to reduce the software development life cycle (SDLC) and improve the effectiveness of building systems by reducing costs (time, efforts and resources) in a range of business domains. Whilst middleware solutions have traditionally been used in business applications, including asset management systems, enterprise management resource planning and e-commerce reservation systems; they rapidly have become in dominant use for Distributed Sensor Actor Networks systems that are built on evolvable, self-healing non-in-situ networks with actuation and control. This encapsulates monitoring and control processes, operations, networks and hardware systems in telecommunications, defense and infrastructure industries.

SANET applications possess distinct characteristics relating to its mission critical aspects and time constraints. Time criticality and strict deadlines is essential, as the correct data response that is delivered beyond a given threshold can result in unpredictable or catastrophic consequences. Therefore, the need for SANET middleware models to meet stringent Quality of Service (QoS) qualitative requirements such as scalability, robustness, usability, security, efficiency, latency, privacy and trust [14][3]. For all application domains, the ultimate goal of middleware is to support the process of software development by facilitating integration of components and protecting engineers from inherent and accidental complexities related to heterogeneous computing and systemic environments.

Chris Chiu, Zenon Chaczko and Perez Moses are with the Faculty of Engineering \& IT, University of Technology, Sydney, Australia (E-mail: christopher.chiu@uts.edu.au, zenon@eng.uts.edu.au, zindpm@gmail.com)
As part of the Cooperative Oriented Sensors Actuators Network model (COSA) proposed by Chaczko et al [5], the modeling of a SANET neighborhood as a local abstraction is moderated by two global aspects: the accuracy of a node's position and the node's immunity to error propagation. The algorithmic categories are used for an adaptive SANET environment, listed from local to global domain concerns:

\section{Feature Mapping}

By using an Extended Kohonen Map (EKM) proposed by Kohonen [11][12], the self-organizing map partitions sensory spaces into discrete regions; thus the map's generalization capability arises from its self-organization during training [13]. Therefore, the sensory representation's resolution is improved in frequently encountered stimuli regions, reflecting biological sensory perceptions where frequent practice leads to prediction of anticipated events.

\section{Multi-variate Regression Techniques}

The task can be formulated as a non-linear multi-variate regression problem [8][9][10]. However, the main concern is that training samples must be collected regularly to define error signals. The sampling process is simplified by providing qualitative feedback at the end of the executing control sequence [10]. The evaluation of using this technique is to ensure the smoothness of when local minima or maxima results are encountered, such that variations are minimized when the target is reached.

\section{Multi-Objective Meta-Heuristics}

Reinforcement learning heuristics are inspired from biological concepts to determine an optimum solution to complex problems. Determining an optimum routing and clustering organization needs adaptation depending on the situation and context [4]. Therefore, this closes the feedback loop to ensure that knowledge from the environment is retained, so predictive models can determine future clustering and routing paths can better model system state behavior.

The unifying characteristic of modern middleware platforms, for project development work and SANET applications, is the paradigm of common data repositories that can be accessed concurrently, within the existing domain concerns and constraints.

\section{A. Theoretical Principles of Holonic Architectures}

Modern component middleware systems [14][1] allows reusable services to be composed, configured, integrated and installed to build software applications efficiently and reliably, while adhering to needs of distributed shared memory across disparate environments. As shown in Fig. 1, distributed data space concerns are addressed through Tuple Space [2] implementations supported in modern component middleware systems, following the multi-verse concepts of elemental slicing of a core entity of representing the structures and 
interconnections between internal entities [1]. This provides users with a specific set of capabilities:

\section{Connector Facilities within Components}

Includes remote procedure calls, remote method invocation or message passing mechanisms.

\section{Horizontal Models of Infrastructure Services}

Request brokers or publish-subscribe mechanisms between components within the same platform.

\section{Vertical Models of Domain Paradigm}

Common semantics and context awareness, and high-level reusable services from transaction and lease support, to multilayer security for multiple platforms.
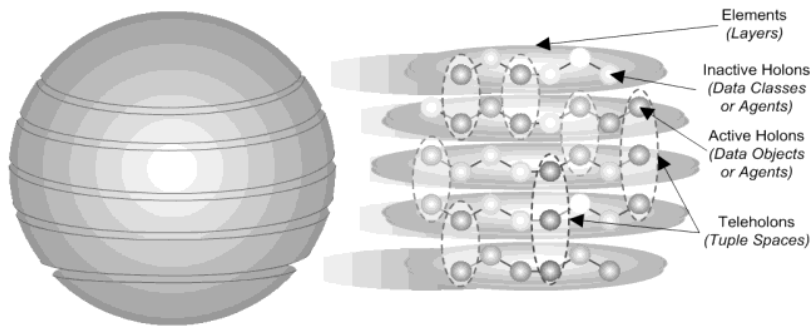

Representation of Core Entity Representation of Sliced Elements

Fig. 1. Tuple Space paradigm consists of distributed Memory Spaces overlayed on multiple platforms [1]

As shown in Fig. 2, the biomimetic model perspective can be projected onto a three-dimensional space, according to the POE Classification Model [1][2]. The POE model represents the different organizational levels of organization, with POE standing for Phylogeny, Ontogeny and Epigenesis.

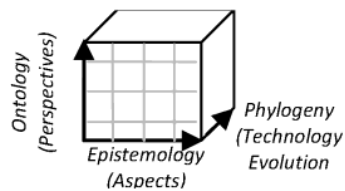

Fig. 2. POE Classification Model (Phylogeny, Ontology, Epistemology) [2]

\section{Phylogeny}

Biological Context: Entail evolution of species genetics

SANET Correlation: This relates to the implementation of heuristic problem solving algorithms (Cooperative EKMs) and the evolution of technology.

\section{Ontogeny}

Biological Context: Concerned with cellular growth process, multi-cellular organization, cellular division and differentiation from the parent to child cells. Each child cell processes a copy of the original genome.

SANET Correlation: The perspective of the domain space and calculation of EKM activation energies.

\section{Epigenesis}

Biological Context: This involves the adaptation and learning processes. The nervous, immune and endocrine systems are characterized by epigenesis.

SANET Correlation: This space corresponds to the facets or aspects of knowledge acquisition by responding to stimuli and weights adaption feedback.

\section{DOMAIN ANALYSIS OF SANETS}

\section{A. Co-operative Extended Kohonen Maps (EKMs)}

For all nodes in a SANET's control space, procedural controls are formed as a discrete set of commands to be used by reinforcement learning algorithms [12] to be selected from a library of heuristic functions. Autonomous systems theory and reinforcement learning are driven by continuous control space functions [11], as indirect-mapping methods provide finer decision choices than in direct mapping. The accuracy in sensory stimuli control is important where external factors directly affect the network's robustness and reliability. The development of a feature map approach using co-operative EKMs with indirect mapping assists to improve the responsiveness of the mapping process [4][11]:

\section{By Direct Mapping Method}

Map inputs of a node directly to sensory stimuli. Indirectmapping approach maps sensory stimuli indirectly to a node with the utilization of control parameters, including energy discharge, signal strength and node roles as ordinary node, cluster head (routable to sink) or precise anchors (GPS nodes).

\section{By Indirect-Mapping Method}

Map the continuous sensory stimuli space to the node; as opposed to direct-mapping that map the continuous sensory stimuli space to discrete directives. Quality evaluators determine optimum clustering using Calinski-Harabasz and Davies-Bouldin [5] criteria.

Traditionally, predictive event capability can be achieved using linear trajectory models in Euclidean space [10]. However, addressing multi-dimensional problems that are outside of the Euclidean space cannot be mapped in isolation, such as network interference and ensuring energy efficiency. This is because it does not factor the entire domain concern of including the problem set into a singular processing model.

Therefore, it is intuitive to convert these dimensional views and decompose them into a singular map of the highest activation energies or stimuli, which is achieved once the maximum value of the integration matrix is calculated for all EKMs for each of the input stimuli received from the SANET's environment. Sensory controls that are insufficiently clarified can result in unexpected or undesirable outcomes, leading to a potential difficulties to navigate a route to the gateway [11][12]. When EKMs are established in the weighted-sum ensemble, a similar problem of un-routable outcomes also takes place. This can be solved by using an indirect EKM mapping method in conjunction with multivariate regression, whereby the control vector is manipulated indirectly via a control parameter space [11].

The environmental concern shown in Fig. 3. Co-operative EKM Process [4] for a given SANET domain can be summarized in the following statement tasks:

- For initial state described by input vector u(0) in input space $\mathrm{U}$, inclusive of the entire sensory perception;

- Adapt new sequence of control vectors $\mathrm{c}(\mathrm{t}), \mathrm{t}=0, \ldots, \mathrm{T}-1$, in sensor control space $\mathrm{C}$ and calculate activation energies;

- With resultant goal state elaborated by $u(T) \in U$ that adapts the network structure for a desired objective, thus reacting to the stimuli and changing the input state. 
This problem will be approached by using a multi-objective approach with co-operative EKMs as the modeling function,

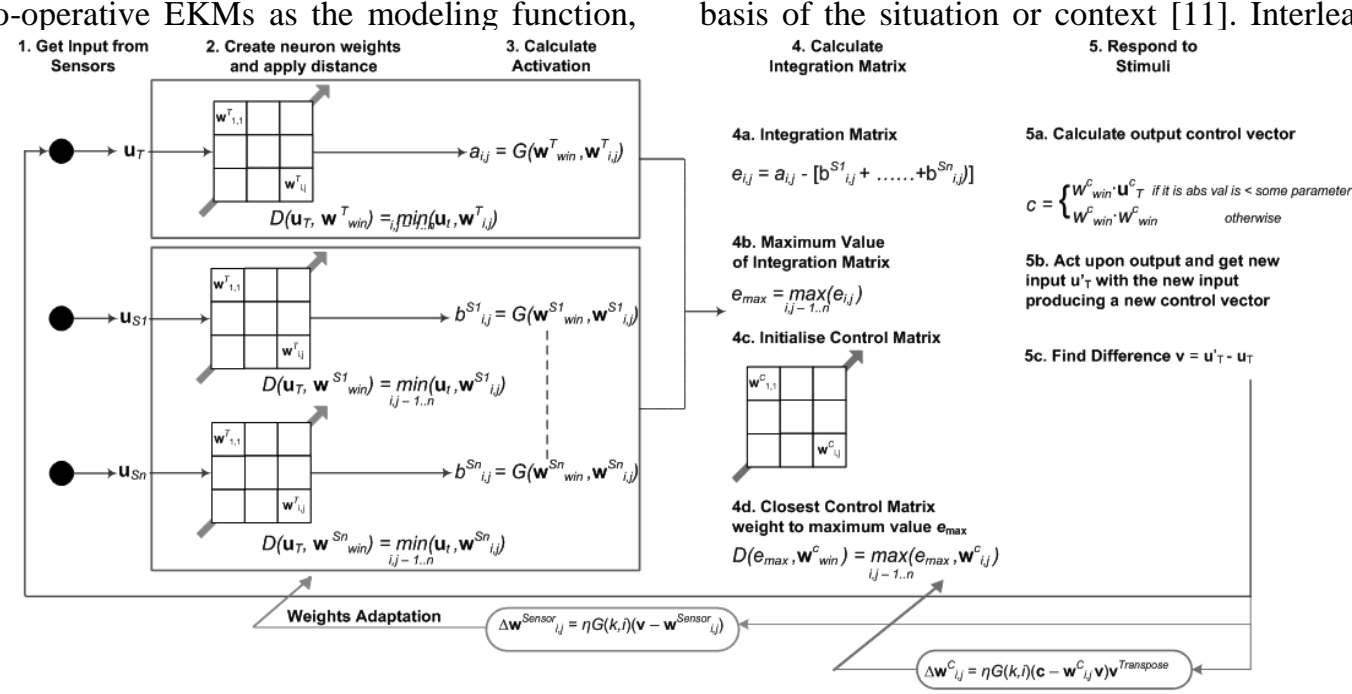

Fig. 3. Co-operative EKM Process [4] with reinforcement learning technique that is chosen on the basis of the situation or context [11]. Interleaved EKMs that cooperate and compete to self-organize can enable a node to optimize in managing clustering and routing for a dynamic environment, whereby the node's output control is less than the total variable control available. In contrast, Euclidean and the weighted-sum ensemble methods reach detrimental outcomes, even though the SANET may implement a continuous sensory control space as proposed by Low [10].

\section{B. Multi-Objective Meta-Heuristic Algorithm (MMA)}

The field of meta-heuristics is about overlaying different heuristic methods to yield an optimal result. In particular, nature-inspired functions are most suitable when considering the learning capability of the SANET network. This is because unlike trajectory functions, which are concerned with only local thresholds to yield a result, nature inspired functions take a more holistic view of the dynamic, evolving system. In essence, by combining a multi-dimensional approach to analyzing a given dataset, we can enhance the regenerative learning capability for any given heuristic optimization model. Current experiments for SANET modeling examine genetic algorithms and Particle Swarm Optimization (PSO) [6], as they both implicit-based reinforcement learning methods that attempt to seek a long term advantage through a representation set of scalar rewards.

The application of the heuristic algorithm follows the approach of applying EKM principles to the problem domain in SANETs [5][6]. This requires the modeling map to be translated into a training vector to be processed against a scalar rewards vector, which reflects the optimum energy or routing condition; depending on the current environmental concerns of the SANET network. The number of evolutions or epochs to determine an acceptable result will affect the quality of the process, but there requires a balance between obtaining a quality evaluation, which requires more processing time and is power intensive, with minimizing the time to process the heuristic function for resource conservation. The implementation method for meta-heuristic functions in the experimental model is established procedurally as shown in Fig. 4. Multi-Objective Meta-Heuristic Process [4].

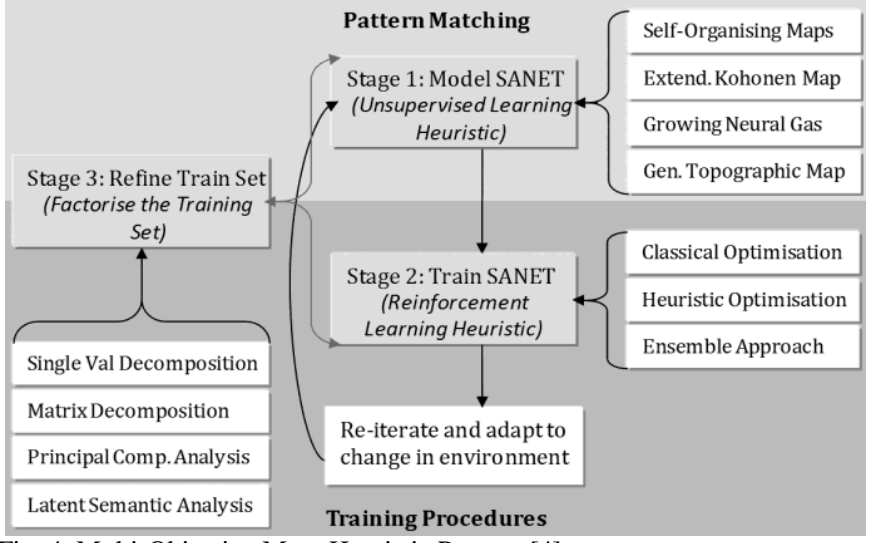

Fig. 4. Multi-Objective Meta-Heuristic Process [4]

- Stage 1: Model Domain Environment (Phylogenical)

This stage is about performing an Unsupervised Learning Heuristic on the environment. This involves modeling the environmental concerns to determine how the data should be organized for optimum clustering and routing mechanisms.

The current experiment considers the use of EKMs to model the SANET environment from the input sensory perception. This process is autonomous and occurs at the beginning of the analysis of the SANET domain.

\section{- Stage 2: Train for Environment (Ontological)}

This stage is about performing a Reinforcement Learning Heuristic. This allows the SANET environment to be aware of its context, and learn from its own experience and that of its cooperative actors.

The experiment incorporates genetic algorithmic heuristics to achieve learning capability. A classical technique such as brute-force analysis is computationally inefficient, and it is not feasible to consider all outcomes that lead to an optimum solution, only the most suitable in the current state in time.

- Stage 3: Refine Training Set (Epigenetical)

This stage is about factorizing the Training Set. This 
optimizes the training set for a given learning heuristic to a singular vector, reducing invariability for long-term forecasting.

The current experiment considers general matrix decomposition techniques to reduce noise and other variability in the training set. This is necessary to select an ideal candidate that establishes the fitness condition for the SANET environment, such as maintaining the minimum energy condition and optimizing available network bandwidth.

\section{EXPERIMENTAL MODEL}

The evaluation of co-operative EKMs on SANETs is executed using the MATLAB environment originally conceived by Chaczko, et al [4]. The simulation framework, shown in Fig. 5. MATLAB Simulation Environment [5] allows for convenient monitoring and tracking of SANET events by pre-assigning event trajectories in the network field.

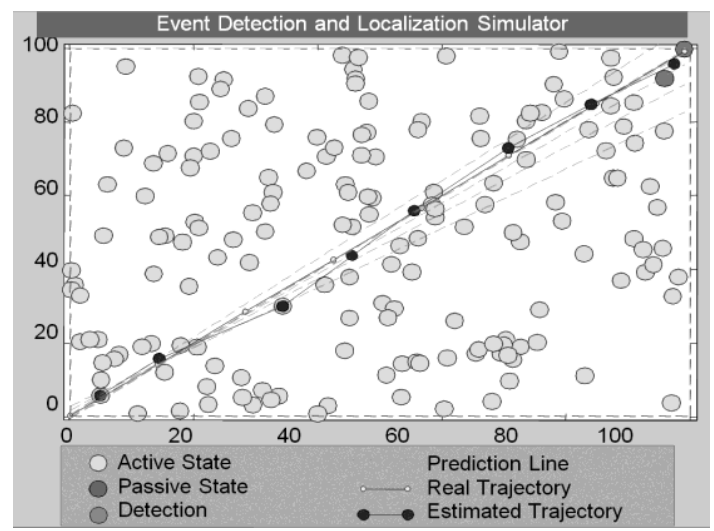

Fig. 5. MATLAB Simulation Environment [5]

\section{A. General Assumptions}

- The topology of the SANET network is two-dimensional, such that altitude is constant.

- All nodes are powered at $100 \%$ energy capacity. Energy dissipation is calculated uniformly using the inverse square law.

- The event trajectory is not predefined, such that the beginning and end points are calculated randomly using the Fast Mersenne-Twister method.

- The network is considered to be interference free, such that RF communication concerns are not evaluated in the experimental model.

\section{B. Experimental Procedure}

The experiment is completed with the methodology by establishing the following simulation constraints:

1. A population of $n$ nodes is distributed randomly via Fast Mersenne-Twister, in a two-dimensional network of 100m x $100 \mathrm{~m}$. Sample node populations include:
a. 100 ;
d. 1000 ;
g. 4000
b. 250
e. 2000
h. 5000 .
c. 500 ;
f. 3000 ;

2. An event trajectory is executed from a point in the network area; of which the test path course is either: a. Linear Path: A linear path consists of an event trajectory where the entry and exit point from the area is of constant gradient.

b. Arc-formation Path: Arc-formation consists of an event trajectory where the entry and exit point will either be increasing or decreasing in gradient, forming a circle segment.

c. Pseudo-random Path: A pseudo-random path using the Mersenne-Twister method combines 2(a) and 2(b) at various points throughout the trajectory, until it reaches the exit point.

3. The algorithm selects the route from the node in range of the approximate trajectory to be established to the sink; such that the closer the algorithm is to calculating the event path, the more optimum the route will be to establish successful communications to the sink.

a. Linear Approximation: Linear approximation estimates the approximate trajectory to determine the route path with Euclidean geometry to calculate the final point of the event, based on the current nodes that are in contact with the event and tracing a path between the previous and current nodes.

b. Co-operative EKMs with PSO: Co-operative EKMs use an indirect-mapping SOM map to train the control parameters in which to converge at the final trajectory point; in such a fashion to actively train the neural network to seek positive outcomes to determine a route from the trajectory's path to the sink.

In conjunction, the reinforcement learning heuristic applied to the SOM map as a training vector set, and a vector test set is applied to maintain accuracy of path estimation. Using Particle Swarm Optimization [6], the shortest Euclidean distance between the actual trajectory point and the nearest neighboring node is nominated within the routable path to the sink.

4. The experiment is executed for 1000 iterations to calculate the mean rate of successful identification of the trajectory's target point, when the simulated event exits the network area:

a. A maximum margin of error is a $2 \mathrm{~m} \times 2 \mathrm{~m}$ area where a final approximate point is determined.

b. A successful identification is where the final end-point is within a $98 \%$ confidence interval of the entire network. Any other estimation outside of this threshold region is identified as a failed identification and is rejected.

\section{EXPERIMENTAL RESULTS}

The results demonstrate that in comparison to linear approximation and co-operative EKMs with PSO, the final results show there is potential when pseudo-random trajectory tracking is required. While linear approximation of pseudorandom trajectories are expected to perform inadequately as it responds best to constrained, predictable local minima conditions; co-operative EKMs demonstrate a perceptible improvement in the identification rate over linear 
approximation. This is evident with a greater node population, as the granularity of determining a nearest neighboring node to route is reduced for the fixed size of the network.

As shown in, there is approximately a 2-fold improvement in detection rates for the pseudo-random trajectory compared to linear approximation. This indicates that while the cooperative EKM with PSO model shows promise, there is more work to be done to improve and make a more consistent prediction mechanism. Current prediction rates are insufficient to ensure long-term routing conditions, which indicate the process of refining the training set for remodeling is necessary to improve the node routing capability.
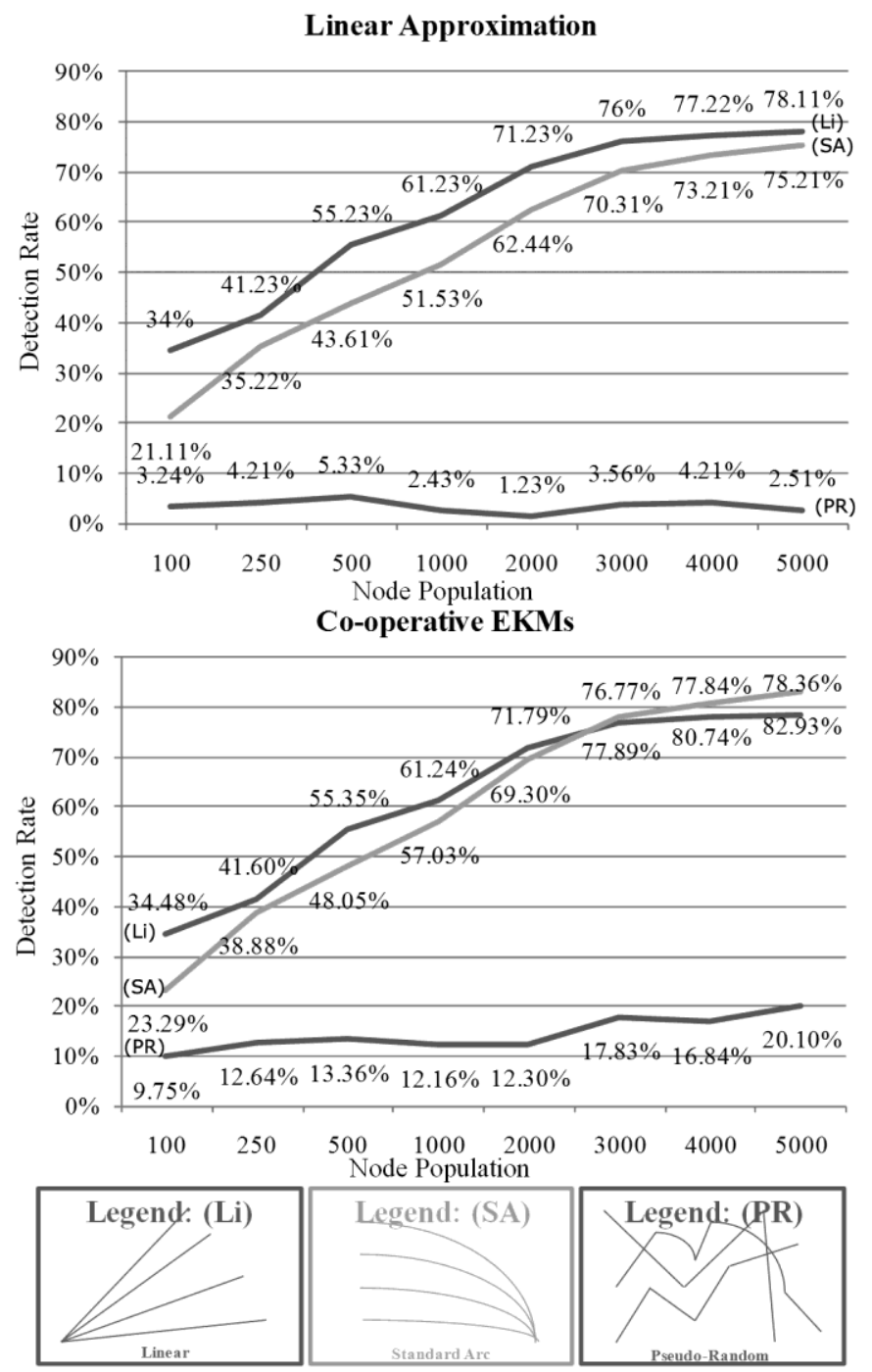

Fig. 6. Experimental Results of Detection Rates

The analysis of co-operative EKMs as shown below in Table $1 \& 2$, when assessed in terms of performance of pseudo-random tracking, requires more analysis into the algorithmic procedure. In particular, the thresholds established for determining positive or negative learning reinforcement is an issue that needs to be evaluated for an in-depth assessment. The tolerance levels used to calculate the thresholds is important, as subtle variations in tolerance may yield undesirable results. As a case in point, reducing tolerance levels too far will result in the inflexibility of the algorithm to adapt to changes the event trajectory; the corollary is that generous tolerance levels will yield undesirable tracking results when noise or faulty nodes produce invalid sensory data.

TABLE $1 \& 2$

COMPARATIVE ASSESSMENT AND EVALUATION

\begin{tabular}{|c|c|}
\hline \multicolumn{2}{|r|}{ LINEAR APPROXIMATION } \\
\hline Descriptor & Elaboration/Observation \\
\hline $\begin{array}{l}\text { Quantitative } \\
\text { Assessment }\end{array}$ & $\begin{array}{l}\text { - Linear approximation is limited with small } \\
\text { node numbers as the sparse distribution is a } \\
\text { poor fit to the estimated Euclidean } \\
\text { geometry path. } \\
\text { - Linear approximation converges to local } \\
\text { minima or maxima too rapidly that prevents } \\
\text { a consistent prediction or targeting of the } \\
\text { final event trajectory }\end{array}$ \\
\hline $\begin{array}{l}\text { Qualitative } \\
\text { Assessment }\end{array}$ & $\begin{array}{l}\text { - Linear approximation cannot compensate for } \\
\text { path obstruction or unpredictable } \\
\text { movement without further algorithmic } \\
\text { improvement. }\end{array}$ \\
\hline
\end{tabular}

CO-OPERATIVE EKMS WITH PSO

\begin{tabular}{cc}
\hline \hline Descriptor & Elaboration/Observation \\
\hline Quantitative & $\begin{array}{c}\text { Demonstrates an indirect-mapping EKM can } \\
\text { provide detection to optimize for local } \\
\text { obstruction and global target identification } \\
\text { concerns. }\end{array}$ \\
Qualitative & $\begin{array}{c}\text { Preliminary results show a smoother tracking } \\
\text { mechanism to monitor events in real-time, } \\
\text { compensating for random events [1] [13]. } \\
\text { Co-operative EKMS with PSO, in conjunction } \\
\text { with an enhanced training set refinement } \\
\text { mechanism, will yield more consistent } \\
\text { results as due to an optimal training vector. }\end{array}$ \\
\hline \hline
\end{tabular}

As a consequence, the potential of Co-operative EKMs to identify events within a SANET network is evident; but as with all passive learning heuristic methods, a heuristic ensemble approach using MMA is necessary to train the algorithm to evaluate and determine the tolerance thresholds that are most suitable for the current conditions. The implementation of co-operative EKMs with alternative heuristic algorithms such as genetic algorithms will need to be considered in future to evaluate improvement in the mean identification rate. Furthermore, a feedback mechanism incorporating training set factorization is required to minimize error rates and ensure a consistent set of data outcomes, and allow for more fluid responses in the routing and tracking network functions.

\section{ANALYSIS OF RESULTS}

\section{A. Hybrid Holonic Architectural Domain Mapping}

The consideration of the Holonic Hybrid architecture corresponds with the needs of the SANET middleware infrastructure, with holons represented as individual resource concerns of which the holonic architecture encapsulates the pool of shared resources for the entire network, and the teleholons represented as the collaboration or synchronization 
between resource holons. As such, it addresses SANET management from two central views:

- Holonic Concerns: Horizontal perspectives for a single cluster; such as the energy conservation task of ensuring optimal sizes for intra-cluster management.

- Telonic Concerns: Vertical perspectives for clusters and external networks, where extra-cluster interaction can facilitate and negotiate common or shared resources that require handover or synchronization.

\section{B. Cooperative EKM Heuristic Validation}

The notable variability in the identification rate indicates a need to improve the quality of training mechanisms to reinforce positive selection processes, so the aggregation of the final routing selection is optimal for the system environment. Furthermore, the implementation of refining the training set is necessary to ensure that the SANET modeling functions do not introduce internally generated erroneous data that will impact on the system's ability to make future predictions based on historical trends.

The current yield in the trajectory projection is insufficient to make meaningful estimations, as the current result is outside the upper 2 standard deviations required to ensure a $95 \%$ confidence interval in accurate tracking projections. This indicates that further work is required before an assessment can be modeled on physical test-bed environments; as realworld scenarios need to be quantitatively evaluated on more stable and consistent system models.

In particular, further examination is required to determine the trade-off between yielding an efficient route which will be more computationally intensive, or a best-effort route that will be more energy conservative. However, the distributed nature of SANETs means that computational calculations can take place for each routing hop, so that while the local calculations are considered for each route, a global picture can be established for the entire route back to the sink or gateway.

\section{CONCLUSION}

The method of adaptive SANET management with cooperative EKMs coupled with multi-objective heuristic functions show promise, with the current results demonstrating indirect-mapping EKM generating more proficient routing and clustering conditions when compared to direct-mapping EKMs and trajectory functions. Furthermore, the control parameters of the indirect-mapping EKM can be trained with recursive least squares to allow convergence and better optimization, with choices in the reinforcement learning technique providing context-aware outcomes to optimize the reinforcement learning capability of the SANET management.

\section{ACKNOWLEDGEMENTS}

We acknowledge the contribution of Michal Nikodem for his prior development of the MATLAB simulation framework to assess the viability for Extended Kohonen Maps in wireless sensor networks.

\section{REFERENCES}

[1] Chaczko, Z. et al, (2005), A Bio-inspired Telecollaboration Service Taxonomy: Usability Related Concerns, Broadcom 2008

[2] Chaczko, Z. et al, (2007), NIE Models of Biomimetic Software Systems, CSIRO ICT Centre Conference.

[3] Chaczko, Z., Davis, D., Mahadevan, V. (2004), New Perspectives on Teaching and Learning Software Systems Development in Large Groups, IT Higher Education \& Training 2004, Istanbul, Turkey

[4] Chaczko, Z; Chiu, C. (2009), Cooperative EKMs for Wireless Sensor Networks, Eurocast 2009, Vol.12, pp. 304-305, published by Gran Canarias Las Palmas University Press

[5] Chaczko, Z; Nikodem, J; Klempous, R; Nikodem, M; Sensors Localisation Methods in the COSA Framework (2007), AusWireless: The 2nd International Conference on Wireless Broadband and Ultra Wideband Communications, pp. 60

[6] Clerc, Maurice (2006), Particle Swarm Optimization: ISTE (International Scientific and Technical Encyclopedia), translated from L'optimisation par essaims particulaires. Versions paramétriques et adaptatives, Hermès Science, 2005.

[7] Ghosh, J. and Nag, A. (2001). An overview of radial basis function networks. In R. J. Howlett and L. C. Jain, editors, Radial Basis Function Networks: New Advances in Design, pp. 1-36. Physica-Verlag, New York

[8] Kaelbling, L. P., Littman, M. L., and Moore, A. W. (1996) Reinforcement learning: A survey. J. Artificial Intelligence Res., pp. 237-285

[9] Kohonen, T. (2000). Self-Organizing Maps. Springer, New York, 3rd Edition.

[10] Low, K. H., Leow, W. K., and Ang, Jr., M. H. (2004). Task allocation via self organizing swarm coalitions in distributed mobile sensor network. In Proc. 19th National Conference on Artificial Intelligence (AAAI-04), pp. 28-33

[11] Low, K. H., Leow, W. K., and Ang, Jr., M. H. (2005), An Ensemble of Cooperative Extended Kohonen Maps for Complex Tasks In Neural Computation, Vol. 17, Issue 6, pp. 1411-1445, published by MIT Press

[12] Mill'an, J. del R., Posenato, D., and Dedieu, E. (2002). Continuousaction Qlearning. Machine Learning, pp. 249-265.

[13] Sharkey, A. J. C. and Sharkey, N. E. (1997). Combining diverse neural nets. Knowledge Engineering Review, pp. 231-247.

[14] Szyperski, C. (1998), Emerging component software technologies - a strategic comparison, Software - Concepts and Tools, Vol 19, No 1, p210 . 